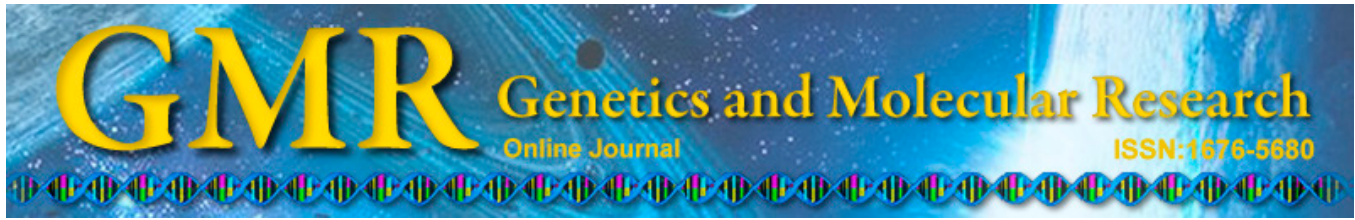

\title{
Upregulation of salivary $\alpha 2$ macroglobulin in patients with type 2 diabetes mellitus
}

\author{
J.K. Feng*, Y.F. Lu*, J. Li, Y.H. Qi, M.L. Yi and D.Y. Ma \\ Center of Laboratory Medicine, \\ The Affiliated Yuhuangding Hospital of Qingdao University Medical College, \\ Yantai, China \\ *These authors contributed equally to this study. \\ Corresponding author: J. Li \\ E-mail: fengtao7668@163.com
}

Genet. Mol. Res. 14 (1): 2268-2274 (2015)

Received March 24, 2014

Accepted July 28, 2014

Published March 27, 2015

DOI http://dx.doi.org/10.4238/2015.March.27.12

\begin{abstract}
We investigated the expression of salivary $\alpha 2$ macroglobulin $(\alpha 2-\mathrm{MG})$ in patients with type 2 diabetes mellitus (T2DM) to investigate its value for predicting damage to the salivary glands. A total of 116 patients with T2DM and 60 patients with impaired fasting glucose (IFG) were included in this study. Sixty health volunteers were enrolled as a control group. Unstimulated saliva was collected at 8 a.m. prior to breakfast. Expression of $\alpha 2-\mathrm{MG}$ was determined using an enzyme-linked immunosorbent assay. The correlation between salivary $\alpha 2-\mathrm{MG}$, serum $\alpha 2-\mathrm{MG}$, and concentration of fasting glucose was analyzed using Pearson correlation analysis. No significant difference was observed in the expression of serum $\alpha 2-\mathrm{MG}$ in the T2DM group, IFG group, and control group ( $\mathrm{P}>0.05$ ). Compared with the control group and IFG group, a statistical difference was observed in the salivary $\alpha 2-\mathrm{MG}$ in the T2DM group $(\mathrm{P}<0.01)$. No statistical difference was observed in the salivary $\alpha 2-\mathrm{MG}$ in the IFG group compared with the control group $(\mathrm{P}>0.05)$. In the patients with T2DM, a close correlation was identified in the expression of serum $\alpha 2-\mathrm{MG}$ and salivary $\alpha 2-\mathrm{MG}(\mathrm{r}=0.52, \mathrm{P}<0.01)$. A poor correlation was
\end{abstract}


identified between salivary $\alpha 2-\mathrm{MG}$ and blood sugar level $(\mathrm{r}=-0.12$, $\mathrm{P}=0.199$ ). The expression of salivary $\alpha 2-\mathrm{MG}$ showed a remarkable increase in T2DM patients, which may be associated with functional disorders of the salivary gland.

Key words: $\alpha 2-\mathrm{MG}$; Saliva; Salivary gland; Type 2 diabetes mellitus

\section{INTRODUCTION}

Type 2 diabetes mellitus (T2DM) is a chronic non-communicable disease caused by the decline of human islet function. The main clinical manifestation in patients with T2DM is high blood sugar. In general, a series of complications, including retinopathy, nephropathy, neuropathy, cardiovascular disease, peripheral vascular disease, and periodontitis have been reported in these patients (Agarwal et al., 2012). Currently, oral infection such as periodontitis and tooth loss has also been reported to be associated with the occurrence of T2DM (Border et al., 2012; Chan et al., 2012). In addition, salivary flow and composition in diabetic patients showed a significant difference compared to that in non-diabetic subjects (Bajaj et al., 2012; Lasisi and Fasanmade, 2012). In particular, significant differences were noted in the expression of salivary proteins in diabetic patients compared with non-diabetic groups (Rao et al., 2009).

Adequate flow of saliva into the oral cavity is very important for maintaining oral health. Reduced saliva output may have a negative effect on dental quality and oral infections. For example, xerostoma has been frequently cited as a symptom of diabetes. Therefore, changes of saliva output and the expression of salivary proteins may indicate altered salivary gland function. In this study, the expression of human $\alpha 2$ macroglobulin $(\alpha 2-\mathrm{MG})$ was determined in T2DM patients. In addition, the correlation between serum $\alpha 2-\mathrm{MG}$, salivary $\alpha 2-\mathrm{MG}$, and fasting blood sugar was analyzed to investigate the predictive effects of salivary $\alpha 2-\mathrm{MG}$ for diagnosing salivary gland disorder.

\section{MATERIAL AND METHODS}

\section{Patients}

A total of 116 T2DM patients (male: 54, female: 62 , average age of $57 \pm 12.3$ years) were treated in the Endocrinology Department and Outpatient Department of Yantai Yuhuangding Hospital from February 2011 to March 2012 and were included in this study. In addition, 60 cases with impaired fasting glucose (IFG, male: 27, female: 33 ) with an average age of $55 \pm$ 14.3 years were enrolled in the IFG group. The diagnosis of T2DM was based on the standards established by the American Diabetes Association in 2010 (American Diabetes Association, 2010). Patients with IFG and a fasting blood glucose $\geq 7.0 \mathrm{mM}$ were considered to be in a prediabetic state. In addition, 60 healthy volunteers (male: 22 , female: 38 , average age of $51 \pm$ 11.3 years) were enrolled as the control group. Fasting blood glucose ranged from 5.6-6.9 mM according to the standards established by the American Diabetes Association in 2003 (International Diabetes Federation, 2003). Those with acute and chronic nephrosis, liver disease, and autoimmune diseases were excluded from this study. All patients signed informed consent. This study was approved by the Ethics Committee of Yuhuangding Hospital. 
Prior to enrolling patients, laboratory testing results and clinical symptoms were used as references for grouping. The onset time of T2DM was more than 4 years. However, because of medical ethics considerations, no limitations were imposed for the clinical treatment during sample collection. Patient information is summarized in Table 1.

\begin{tabular}{lccc} 
Table 1. Patient information. \\
\hline Item & T2DM group & IFG group & Control group \\
\hline Number $(\mathrm{M} / \mathrm{F})$ & $116(54 / 62)$ & $60(27 / 33)$ & $60(22 / 38)$ \\
Age (years) & $57 \pm 12.3$ & $55 \pm 14.3$ & $51 \pm 11.3$ \\
Time of onset (years) & $>4$ & First diagnosis or recheck & NA \\
Tooth loss (N) & 2 & 1 & NA \\
Diabetic nephropathy $(\mathrm{N})$ & 9 & $\mathrm{NA}$ & $\mathrm{NA}$ \\
Diabetic foot $(\mathrm{N})$ & 8 & $\mathrm{NA}$ & $\mathrm{NA}$ \\
Diabetic eye disease $(\mathrm{N})$ & 3 & $6.58 \pm 0.24$ & $\mathrm{NA}$ \\
Fasting blood sugar $(\mathrm{mM})$ & $10.08 \pm 2.44$ & $1.57 \pm 0.36$ & $5.01 \pm 0.41$ \\
Serum $\alpha_{2}-\mathrm{MG}(\mathrm{g} / \mathrm{L})$ & $1.70 \pm 0.55$ & $158.1 \pm 60.1$ & $1.54 \pm 0.38$ \\
Salivary $\alpha_{2}-\mathrm{MG}(\mathrm{ng} / \mathrm{mL})$ & $192.6 \pm 65.3$ & $5.8 \pm 1.1$ & $134.8 \pm 63.2$ \\
HbAlc $(\%)$ & $8.7 \pm 1.7$ & & $5.7 \pm 0.7$ \\
\hline
\end{tabular}

\section{Sample collection and processing}

Unstimulated saliva $(1 \mathrm{~mL})$ from the diabetic and control group was collected. Briefly, at 8 a.m., the subjects were asked to rinse their mouths thoroughly with water prior to breakfast. They were then required to tilt their heads forward, and saliva was collected into a sterile container. The saliva samples were immediately frozen and stored at $-20^{\circ} \mathrm{C}$ until further analysis. Saliva samples were centrifuged at $2000 \mathrm{~g}$ for $10 \mathrm{~min}$ at $4^{\circ} \mathrm{C}$. The supernatants were used for the detection of salivary proteins using an enzyme-linked immunosorbent assay kit (RapiBio, Inco, CA, USA) following the manufacturer instructions.

For serum samples, $3 \mathrm{~mL}$ venous blood was collected from the ulnar vein. Next, the blood was transferred to a vacuum blood tube. After centrifugation at $2000 \mathrm{~g}$ for $5 \mathrm{~min}$ at $4^{\circ} \mathrm{C}$, the samples were immediately frozen and stored at $-20^{\circ} \mathrm{C}$. The concentration of $\alpha 2-\mathrm{MG}$ was determined using a BNII automatic protein analyzer (Siemens, Munich, Germany). The level of blood glucose was detected using aDXC800 automatic biochemistry analyzer (Beckman Coulter, Brea, CA, USA) according to manufacturer instructions.

\section{Statistical analysis}

SPSS16.0 was used for statistical analysis (SPSS, Inc., Chicago, IL, USA). All data are reported as means \pm standard deviation. One-factor analysis of variance was performed for inter-group comparison. The correlation between salivary $\alpha 2-\mathrm{MG}$, serum $\alpha 2-\mathrm{MG}$, and concentration of fasting glucose was analyzed by Pearson correlation analysis. $\mathrm{P}<0.05$ was considered to be statistically significant.

\section{RESULTS}

\section{Expression of serum $\alpha_{2}-\mathrm{MG}$ and salivary $\alpha_{2}-\mathrm{MG}$}

Table 2 summarizes blood glucose concentration, serum $\alpha_{2}-\mathrm{MG}$, and saliva $\alpha_{2}-\mathrm{MG}$ in 
T2DM patients, the IFG group, and the control group. No statistical difference was observed in the blood glucose and serum $\alpha_{2}-\mathrm{MG}$ among groups $(\mathrm{P}>0.05)$. Compared with the control group, a statistical difference was observed in salivary $\alpha_{2}-\mathrm{MG}$ in the T2DM group and IFG group (P< $0.01)$. However, no statistical difference was observed in the T2DM and IFG group $(\mathrm{P}>0.05)$.

Table 2. Comparison between serum $\alpha_{2}-\mathrm{MG}$ and salivary $\alpha_{2}$-MG.

\begin{tabular}{lcccc}
\hline Group & $\mathrm{N}$ & Blood glucose $(\mathrm{mM})$ & Serum $\alpha_{2}$-MG $(\mathrm{g} / \mathrm{L})$ & Salivary $\alpha_{2}-\mathrm{MG}(\mathrm{ng} / \mathrm{mL})$ \\
\hline T2DM group & 116 & $10.08 \pm 2.44$ & $1.70 \pm 0.55$ & $192.6 \pm 65.3^{*}$ \\
IFG group & 60 & $6.58 \pm 0.24$ & $1.57 \pm 0.36$ & $158.1 \pm 60.1^{*}$ \\
Control group & 60 & $5.01 \pm 0.41$ & $1.54 \pm 0.38$ & $134.8 \pm 63.2$ \\
\hline
\end{tabular}

$* \mathrm{P}<0.05$, compared with control group.

\section{Correlation between blood sugar and salivary $\alpha 2-\mathrm{MG}$ in T2DM patients}

Correlation analysis was performed by analyzing the blood sugar level in 116 T2DM patients as an independent variable and salivary $\alpha 2-\mathrm{MG}$ level as a dependent variable. A poor correlation was observed between blood sugar and salivary $\alpha 2-\mathrm{MG}$ in T2DM patients $(\mathrm{r}=$ $0.12, \mathrm{P}=0.199$, Figure 1).

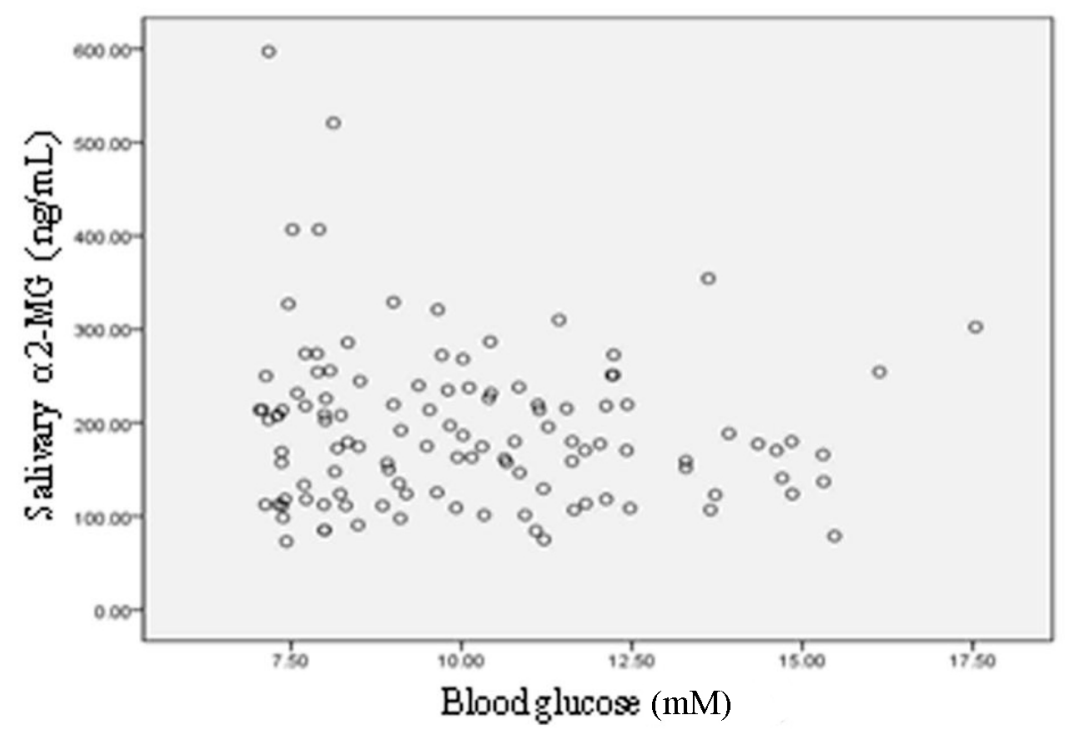

Figure 1. Correlation analysis between blood sugar and salivary $\alpha 2-\mathrm{MG}$ in T2DM.

\section{Correlation between serum $\alpha 2-\mathrm{MG}$ and salivary $\alpha 2-\mathrm{MG}$ in T2DM patients}

For the correlation analysis between serum $\alpha 2-\mathrm{MG}$ and salivary $\alpha 2-\mathrm{MG}$ in T2DM patients, serum $\alpha 2-\mathrm{MG}$ was considered the independent variable, while salivary $\alpha 2-\mathrm{MG}$ level was considered the dependent variable. A significant correlation was observed between serum $\alpha 2-\mathrm{MG}$ and salivary $\alpha 2-\mathrm{MG}$ in T2DM patients $(\mathrm{r}=0.52, \mathrm{P}<0.01$, Figure 2$)$. 


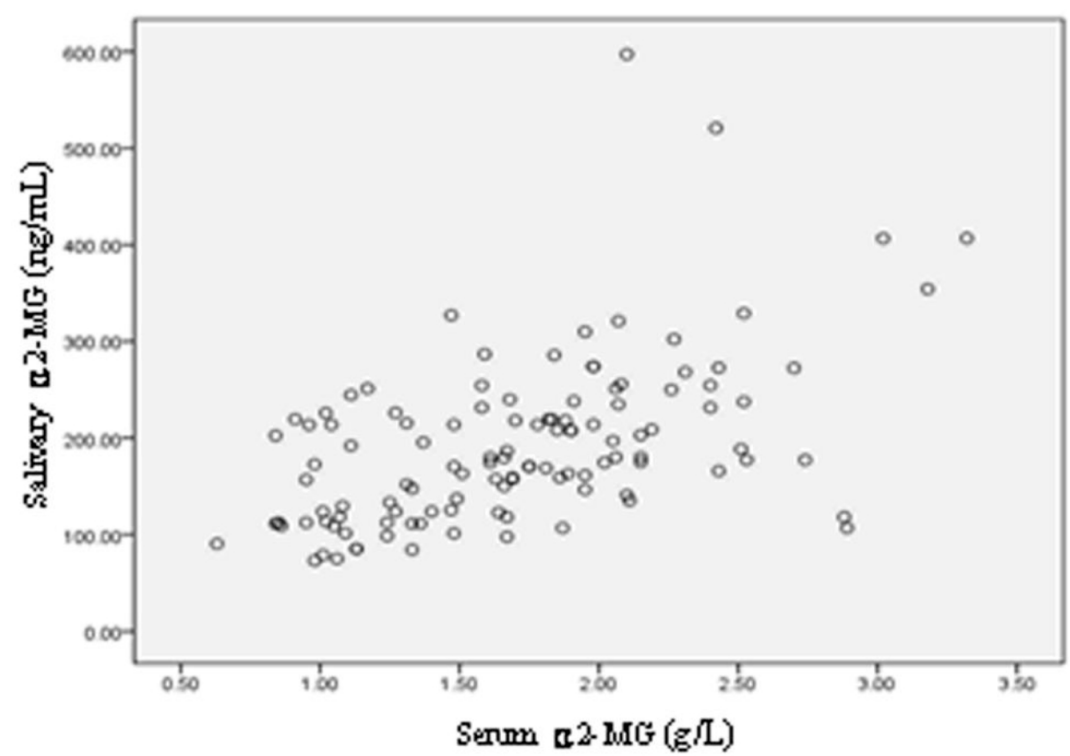

Figure 2. Correlation analysis between serum and salivary $\alpha 2-\mathrm{MG}$ in T2DM.

\section{Correlation between serum $\alpha 2-$ MG and salivary $\alpha 2-$ MG in the control group}

In our study, the correlation between serum $\alpha 2-\mathrm{MG}$ and salivary $\alpha 2-\mathrm{MG}$ in the control group was also investigated using serum $\alpha 2-\mathrm{MG}$ as the independent variable and salivary $\alpha 2-M G$ level as the dependent variable. Our results showed a poor correlation between serum $\alpha 2-\mathrm{MG}$ and salivary $\alpha 2-\mathrm{MG}$ in the control group $(\mathrm{r}=0.182, \mathrm{P}=0.163$, Figure 3$)$.

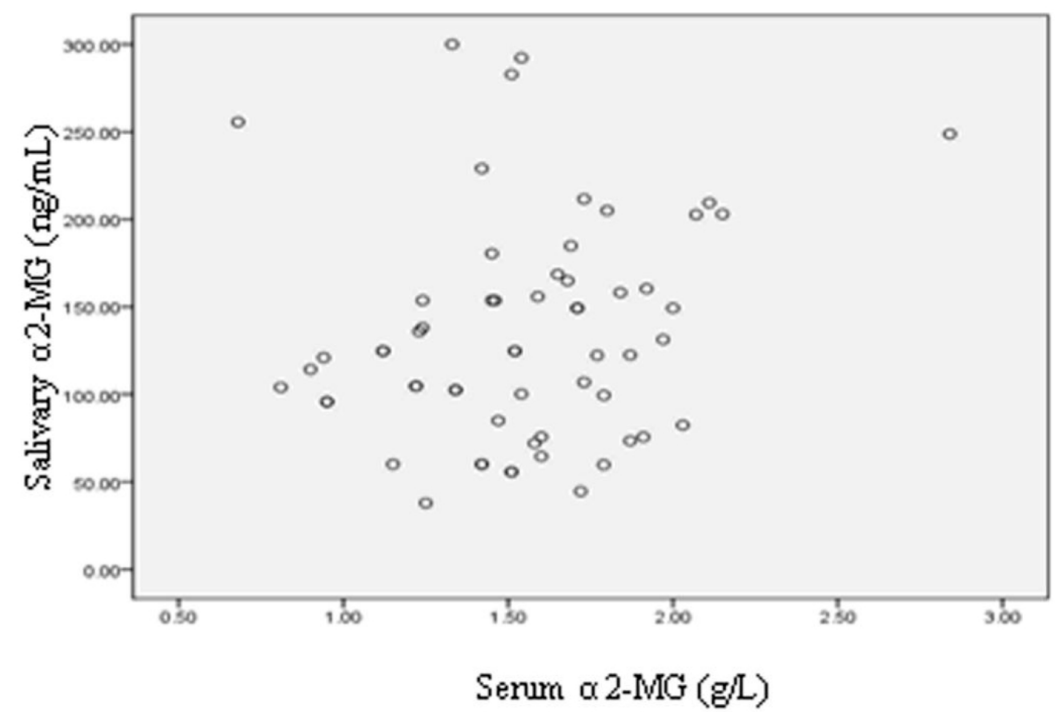

Figure 3. Correlation analysis between serum and salivary $\alpha 2-\mathrm{MG}$ in the normal control group. 


\section{DISCUSSION}

The incidence of T2DM continues to increase each year. Great progress has been achieved in treatment methods, and the therapeutic schedule is rigorous and has been standardized. To date, effective treatment plans for diabetes have improved the quality of life and delayed the incidence of a variety of complications. However, a large number of patients develop chronic complications in elderly years (Agarwal et al., 2012). A recent study indicated that the levels of some salivary proteins such as $\alpha 2-\mathrm{MG}, \alpha 1$-antitrypsin, and chalone $\mathrm{C}$ in patients with T2DM was 2-fold higher than these values in non-diabetic subjects (Rao et al., 2009). These proteins are considered to be associated with glucose metabolism and immunoregulation pathways.

We tested serum and salivary $\alpha 2-\mathrm{MG}$ levels in T2DM patients and IFG patients. The results showed that the serum $\alpha 2-\mathrm{MG}$ in T2DM patients was higher than those in the control group and IFG group. However, no statistically significant difference was noted among serum $\alpha 2-\mathrm{MG}$ in these patients. A previous study (James et al., 1980) reported a remarkable increase in serum $\alpha 2-\mathrm{MG}$ in patients with T2DM, but this was largely influenced by gender and age. In our study, salivary $\alpha 2-\mathrm{MG}$ level was significantly higher than in the normal control group and IFG group, which was consistent with a previous report (Rao et al., 2009). In contrast, Yin et al. (2012) reported that in edentulous patients with T2DM, downregulation of salivary $\alpha 2-\mathrm{MG}$ was observed. We speculated that tooth loss, gingival tissue repair, and diet may affect the secretion of salivary proteins. In a recent study investigating putative local etiologic factors on implant bone loss in relation to T2DM, salivary osteoprotegerin was higher in the diabetes group than in the control group at baseline levels, while the levels of IL-4 and IL-10 in the diabetes group showed a remarkable decrease compared to that in the control group (Tatarakis et al., 2013).

As a poor correlation between blood sugar and salivary $\alpha 2-\mathrm{MG}$ was identified in patients with T2DM, we speculated that the significant increase in salivary $\alpha 2-\mathrm{MG}$ in diabetes patients was not induced by the increased blood glucose concentration. The level of serum $\alpha 2-\mathrm{MG}$ was positively related to salivary $\alpha 2-\mathrm{MG}$ in T2DM patients, but a poor correlation was observed between serum $\alpha 2-\mathrm{MG}$ and salivary $\alpha 2-\mathrm{MG}$ in the normal control group. This indicated that some alternations may occur when $\alpha 2-\mathrm{MG}$ is secreted from the blood into the saliva in T2DM patients. However, the mechanism of how $\alpha 2-\mathrm{MG}$ enters the saliva has not been well defined. Based on our results, the significant increase in salivary $\alpha 2-\mathrm{MG}$ in T2DM patients may be associated with a higher level of serum $\alpha 2-\mathrm{MG}$ and the secretion function of the salivary gland. In addition, alterations in the secretion function of salivary glands may indicate damage to the salivary gland.

Further histopathological examinations are needed to confirm the salivary gland lesion. However, biopsy is more invasive than other methods. In this study, we only collected unstimulated saliva prior to breakfast. Further studies are needed to investigate the effects of stimulated whole saliva and salivary flow on the altered function of the salivary gland.

Most biological markers in the blood can be detected in the saliva. However, their concentrations are low (Denny et al., 2008). The level of certain biomarkers in the saliva changes during the disease course (Hu et al., 2007). Wong (2008) paved the way for the saliva research in 2008, and saliva diagnostics have been extensively applied in molecular diagnostics since.

In clinical experiments, it is easy to collect and store saliva. In addition, the procedure is non-invasive and rapid. Along with the clinical application of sensitive experimental tech- 
nology and equipment, saliva can be used for disease diagnosis. Saliva is important for disease diagnosis and progression monitoring (Greenberg et al., 2010).

In conclusion, the expression of salivary $\alpha 2-\mathrm{MG}$ showed a remarkable increase in T2DM patients compared with the control group. For patients with T2DM, a close correlation was identified in the expression of serum $\alpha 2-\mathrm{MG}$ and salivary $\alpha 2-\mathrm{MG}$. Our study may provide some evidence regarding the association between expression of salivary $\alpha 2-\mathrm{MG}$ and functional disorders of the salivary gland.

\section{Conflicts of interest}

The authors declare no conflict of interest.

\section{ACKNOWLEDGMENTS}

Research supported by the Yantai Scientific Development Program (\#2011205).

\section{REFERENCES}

Agarwal AK, Singh M, Arya V, Garg U, et al. (2012). Prevalence of peripheral arterial disease in type 2 diabetes mellitus and its correlation with coronary artery disease and its risk factors. J. Assoc. Phys. India 60: 28-32.

American Diabetes Association (2010). Summary of revisions for the 2010 Clinical Practice Recommendations. Diabetes Care 33 (Suppl 1): S3.

Bajaj S, Prasad S, Gupta A and Singh VB (2012). Oral manifestations in type-2 diabetes and related complications. Indian J. Endocrinol. Metab. 16: 777-779.

Border MB, Schwartz S, Carlson J, Dibble CF, et al. (2012). Exploring salivary proteomes in edentulous patients with type 2 diabetes. Mol. Biosyst. 8: 1304-1310.

Chan HH, Rahim ZH, Jessie K, Hashim OH, et al. (2012). Salivary proteins associated with periodontitis in patients with type 2 diabetes mellitus. Int. J. Mol. Sci. 13: 4642-4654.

Denny P, Hagen FK, Hardt M, Liao L, et al. (2008). The proteomes of human parotid and submandibular/sublingual gland salivas collected as the ductal secretions. J. Proteome Res. 7: 1994-2006.

Greenberg BL, Glick M, Frantsve-Hawley J and Kantor ML (2010). Dentists' attitudes toward chairside screening for medical conditions. J. Am. Dent. Assoc. 141: 52-62.

Hu S, Loo JA and Wong DT (2007). Human saliva proteome analysis and disease biomarker discovery. Expert Rev. Proteomics 4: 531-538.

International Diabetes Federation (2003). IDF Diabetes Atlas. 2nd edn. International Diabetes Federation, 2013, Brussels, Belgium.

James K, Merriman J, Gray RS, Duncan LJ, et al. (1980). Serum alpha 2-macroglobulin levels in diabetes. J. Clin. Pathol. 33: $163-166$.

Lasisi TJ and Fasanmade AA (2012). Salivary flow and composition in diabetic and non-diabetic subjects. Niger J. Physiol. Sci. 27: 79-82.

Rao PV, Reddy AP, Lu X, Dasari S, et al. (2009). Proteomic identification of salivary biomarkers of type-2 diabetes. $J$. Proteome Res. 8: 239-245.

Tatarakis N, Kinney JS, Inglehart M, Braun TM, et al. (2013). Clinical, microbiological, and salivary biomarker profiles of dental implant patients with type 2 diabetes. Clin. Oral Implants Res. 25: 803-812.

Wong DT (2008). Salivary diagnostics: amazing as it might seem, doctors can detect and monitor diseases using molecules found in a sample of spit. Am. Sci. 96: 37-43.

Yin J, Gao H, Yang J, Xu L, et al. (2012). Measurement of salivary resistin level in patients with type 2 diabetes. Int. J. Endocrinol. 2012: 359724. 